hole into the sub-retinal space, as it was uninterruptedly doing before the operation was performed.

Gonin's operation, therefore, seems to me to achieve success by " excluding" the hole in the retina, to use the term which we apply to pupillary adhesions, not to "occlusion." In the cured case the position of the retinal hole will correspond with the organized choroidal scar which can be recognized with the ophthalmoscope. It would appear to be necessary to determine as accurately as possible before operating in each case to what part of the choroid the retinal hole will correspond when the sub-retinal fluid flows out, and to puncture with the cautery at this point in the overlying sclerotic; the height of the detachment in the region of the hole will presumably be a factor in determining the spot selected for the puncture; the rather free use of a large cautery would increase the chance of success.

A clear apprehension of the modus operandi of Gonin's operation is desirable, and clear thinking may promote further advance in this method of treatment of detachment of retina, which has already achieved so much success. The expression " sealing the hole" seems inappropriate and misleading : " exclusion of the hole " by ring synechia between retina and choroid is perhaps the phrase which will best convey the state of affairs to the ophthalmic surgeon; in using the term he will not conceive of the annular attachment as merely linear at the extreme margins of the hole; a broader area of the circumference is no doubt adherent to the choroidal scar; the surgeon will not need to be reminded that in the presence of the detachment the direction of the current of fluid is from the intra-ocular to the sub-retinal space.

\title{
AN EPIDEMIC OF CONJUNCTIVITIS DUE TO INFECTION WITH BACILLUS FAECALIS ALKALIGENES
}

BY

\section{R. H. BALFOUR BARROW}

LONDON

My inability to find any record of this organism as a cause of epidemic conjunctivitis leads me to report this outbreak. The epidemic occurred recently in the training ship " Exmouth" which is a training ship for boys for the Royal Navy and the Merchant Service. The ship, which is under the control of the Iondon County Council, lies at anchor in the Thames off the north bank of the river about 20 miles from London. She is built 
for a complement of 700 boys but at the time of the outbreak there were only 460 on board. The ages of the boys vary from 11 to 16 years. The water supply to the ship is by pipe from the main town supply.

On September 6, one boy reported sick with " sore eyes " to the sick berth steward who treated the case with a simple boric wash. On September 9, I visited the ship and found nine boys were similarly affected. The appearance of these cases was a general hyperaemia of both bulbar and palpebral conjunctiva with follicles present in the upper fornix along the upper tarsal margin. In all cases except one the lower fornix was free from follicles. No case had follicles on the bulbar conjunctiva. There was a good deal of photophobia and lacrymation, and a small amount of mucopurulent discharge. The condition of these cases can best be described as very similar to the early stage of trachoma.

On questioning the original case he told me that he had only recently joined the ship, and also that two years previously he had had trachoma. This was borne out by the scarring of the upper lids. In view of this history and the suspicious appearance of the nine cases I decided to treat them as early trachoma pending further investigation. They were isolated forthwith and the rest of the ship's company inspected, but careful examination failed to reveal further cases. The following day an inspection produced eight cases, the next day a similar procedure produced 20 cases. A daily inspection was thenceforth the rule and an increasing number were found to be infected. 'The rapid spread of the infection negatived the suspicion of trachoma but as a precaution the old trachoma case was removed from the ship.

With a view to ascertaining the organism present, swabs of the conjunctival sac were sent to the laboratory for examination. The reports read as follows :-

1. "The Gram-negative bacilli which grew in the cultures are all of the same type of organism and belong to the coliform group. We have not yet been able to classify the organism by culture and fermentation tests, but we are carrying out further tests with regard to its pathogenicity for rabbits."

2 . "The coliform organisms isolated from the conjunctival swabs cause no reaction when instilled into the conjunctival sacs of rabbits or guinea-pigs. It has not yet been possible to classify further the organisms except to prove that they are not KochWeeks' bacillus, Morax-Axenfeld bacillus, or B. pneumoniae (Friedlander).

3. "Cultures of the Gram-negative bacillus conform in their growth, characteristics and fermentation reactions to those of bacillus faecalis alkaligenes."

A word now about the causative organism. The bacillus faecalis 
alkaligenes is a Gram-negative motile bacillus, unable to ferment any carbohydrate, producing a strong alkaline reaction in litmus milk, and a brownish growth on potato ; is frequently found in the intestinal canal, and very occasionally infects its host. It usually has the signilicance of a simple saprophyte but some observers state that it may cause an infection in man.

Morphology.-The size and shape of the organism varies a little according to the conditions under which it is grown but usually it may be described as a short rod, 0.5 by 1 to $2 \mu$, occurring singly or in pairs, occasionally in long chains, and provided with peritrichous flagella.

Cultural characters.-Closely resemble B. typhosus in growth. Flourishes on similar media.

Biochemical reactions.- No fermentation of carbohydrate, no proteolytic ferment is formed, and no digestion of serum or gelatine, although growth is accompanied by alkali formation.

Serological reactions. - In blood serum of man in whose intestine the bacillus may be present in large numbers no agglutinins seem to be developed.

Pathogenicity.-Petruschky was able to infect laboratory animals by intraperitoneal but not by subcutaneous inoculation. Evidence of the organism being pathogenic to man and animals is rare.

Distribution in Nature.-Petruschky found it originally in spoiled beer, and it has been found frequently in water. The bacillus is probably widely distributed and is a mere saprophyte. In the human intestine under normal conditions it probably occurs only in small numbers. Trawinski and Gyorgy (1918) found it in 19 per cent. of normal stools, but on the other hand found it in 71 per cent. of the stools of those suffering with a catarrhal condition of the bowels.

As soon as it was realized that all the boys on the ship were likely to be infected a request was sent to the L.C.C. for extra help and a staff of six nurses was sent down. All school and instructional classes were stopped and the ship placed in quarantine. Every infected case was treated three times a day by washing out the conjunctival sac, and, after the final wash for the day, the lids were smeared with ung. hydrarg. ox. flav. (B.P.) The solutions used for irrigation were lotio ac. boric.; lot. ac. boric. $\overline{\mathrm{c}} \mathrm{zn}$. sulph. (Roy. Lond. Ophthal. Hosp.) and lot. hydrarg. perchlor. $1 / 10,000$. The zinc solution caused a good deal of irritation and after a few days was discontinued. Of the two remaining solutions a careful watch was kept to ascertain whether one was more suitable than the other, but the cases seemed to clear up equally quickly. I have since come to the conclusion that the washing out of the conjunctival sac is the essential, and that probably normal saline 
would have been equally efficacious. The average duration for the treatment of each boy was ten days. All washing both of the person and utensils was carried out with running water. The source of the infection was never traced.

Two graphs are attached. No. 1 shows the rapid infection of the ship's company and the equally rapid recovery. No. 2 shows the daily number of admissions from the first day, with, superimposed, the daily number of discharges from the eleventh day. It will be noticed how nearly these two coincide, admissions reaching their
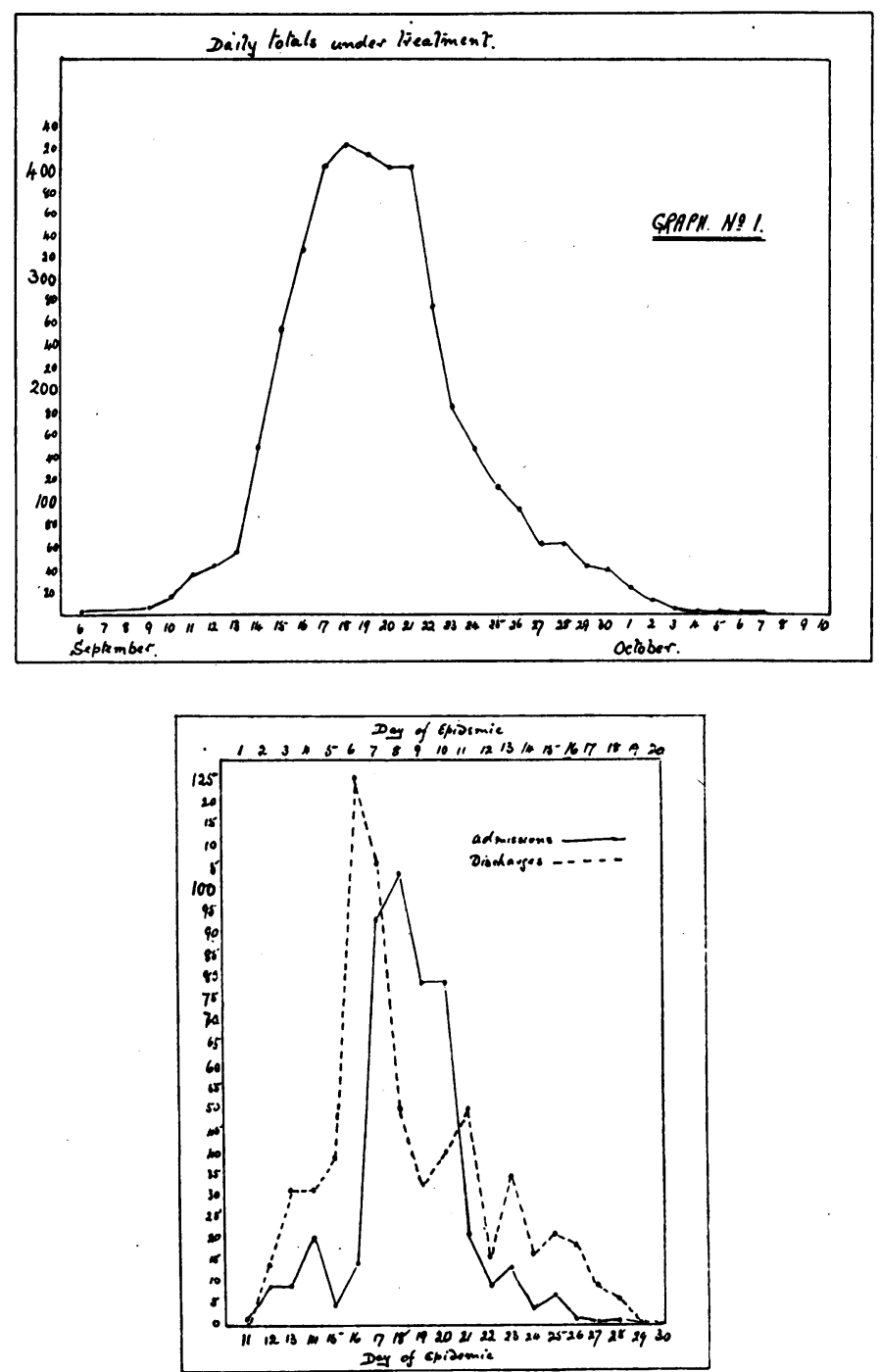
peak on the seventh and eighth days while discharges reached their peak on the sixteenth and seventeenth days.

Lastly, acknowledgments are made to Dr. V. D. Allison for the bacteriological examinations and reports, and to Dr. T. H. Whittington, Consulting Oculist to the L.C.C., who visited the "Exmouth" during the epidemic and approved of what was being done.

\section{GLAUCOMA AND LOW TENSION DISC CUP ENLARGEMENTS}

BY

\section{RANSOM PICKARD}

EXETER

AT the present time some interest is being taken in cases which present all the symptoms of glaucoma except that of increased tension. In April, 1930, I read a paper (a) dealing with this group of cases, showing that, as far as the tests employed were concerned, there was no difference from glaucoma except that of tension. Prof. F. H. Newman, D.Sc., Professor of Physics in the University College of the South-West, afterwards kindly examined the paper and the charts on which it was founded. He agreed that on the facts as presented to him the deductions were correct. He suggested that a plot of the tension against the size of the optic disc cup be made and that the elasticity of the cup should be investigated. The present paper embodies these results, an enquiry into the origin of the low tension cases, compares the clinical course of the two conditions, and discusses the name to be applied to the condition when it occurs with no rise of intra-ocular tension.

The cubic capacity was calculated upon the basis that the transverse diameter of the disc is $1.5 \mathrm{~mm}$., the vertical $1.6 \mathrm{~mm}$. and that a depth of $-3 \mathrm{D}$. equals $1 \mathrm{~mm}$.; the drawings of the discs made in my notes supplying the remaining details. It should be noted that, because of some details being absent, some cases used in the paper ( $a$ ) had to be rejected, while as many of the cases shown in it as " too advanced for use in charts" as possible were added, as the present paper does not deal with the fields of vision, and they are necessary for a more complete comparison of the clinical course.

\begin{tabular}{lccccc} 
& \multicolumn{3}{c}{$\begin{array}{c}\text { No. of eyes } \\
\text { from paper }(a)\end{array}$} & $\begin{array}{c}\text { No. of advanced } \\
\text { eyes added. }\end{array}$ & Total. \\
Glaucoma & $\ldots$ & 32 & 12 & $=$ & 44 \\
Low tension & $\ldots$ & 35 & 2 & $=$ & 37
\end{tabular}

